\title{
Deacetylation of HSD17B10 by SIRT3 regulates cell growth and cell resistance under oxidative and starvation stresses
}

\author{
Lu Liu', Shuaiyi Chen', Miao Yu', Chenxu Ge1, Mengmeng Ren', Boya Liu', Xin Yang ${ }^{1}$, Thomas W. Christian Jr², \\ Ya-Ming Hou', Junhua Zou', Wei-Guo Zhu ${ }^{3}$ and Jianyuan Luo ${ }^{1,4}$
}

\begin{abstract}
17-beta-hydroxysteroid dehydrogenase 10 (HSD17B10) plays an important role in mitochondrial fatty acid metabolism and is also involved in mitochondrial tRNA maturation. HSD17B10 missense mutations cause HSD10 mitochondrial disease (HSD10MD). HSD17B10 with mutations identified from cases of HSD10MD show loss of function in dehydrogenase activity and mitochondrial tRNA maturation, resulting in mitochondrial dysfunction. It has also been implicated to play roles in the development of Alzheimer disease (AD) and tumorigenesis. Here, we found that HSD17B10 is a new substrate of NADdependent deacetylase Sirtuin 3 (SIRT3). HSD17B10 is acetylated at lysine residues K79, K99 and K105 by the acetyltransferase CBP, and the acetylation is reversed by SIRT3. HSD17B10 acetylation regulates its enzymatic activity and the formation of mitochondrial RNase P. Furthermore, HSD17B10 acetylation regulates the intracellular functions, affecting cell growth and cell resistance in response to stresses. Our results demonstrated that acetylation is an important regulation mechanism for HSD17B10 and may provide insight into interrupting the development of AD.
\end{abstract}

\section{Introduction}

17-beta-hydroxysteroid dehydrogenase 10 (HSD17B10), encoded by HSD17B10, is a member of the short-chain dehydrogenase superfamily ${ }^{1}$. HSD17B10 is the only family member located in the mitochondria ${ }^{2}$. It catalyzes the beta-oxidation of fatty acids, androgens, and estrogens, contains 3-alpha-hydroxysteroid dehydrogenase activity, and carries out oxidative conversions of 7-alpha- $\mathrm{OH}$ and 7-beta-OH bile acids ${ }^{3-5}$. HSD17B10 plays an important role in mitochondrial fatty acid metabolism.

Besides mitochondrial dehydrogenase activity, HSD17B10 is also an essential subunit of human mitochondrial ribonuclease $\mathrm{P}$ (RNase P), a complex involved in mitochondrial tRNA maturation ${ }^{6}$. RNase $P$ is a protein complex that cleaves

\footnotetext{
Correspondence: Jianyuan Luo (luojianyuan@bjmu.edu.cn)

${ }^{1}$ Department of Medical Genetics, Center for Medical Genetics, Peking

University Health Science Center, Beijing 100191, China

${ }^{2}$ Department of Biochemistry and Molecular Biology, Thomas Jefferson University,

233 South 10th Street, 220 BLSB, Philadelphia, PA 19107, USA

Full list of author information is available at the end of the article

Edited by N. Bazan
}

tRNA molecules in their $5^{\prime}$-ends ${ }^{7}$. HSD17B10 (MRPP2), together with TRMT10C (MRPP1), forms a subcomplex of the mitochondrial RNase P, called the MRPP1-MRPP2 subcomplex ${ }^{8}$. The MRPP1-MRPP2 subcomplex acts as a tRNA maturation platform. It is involved in the $5^{\prime}$-end cleavage by the mitochondrial RNase $\mathrm{P}$ complex and is responsible for the N1-methylation of adenosine and guanosine at position 9 (m1A9 and $\mathrm{m} 1 \mathrm{G} 9$, respectively) of human mitochondrial tRNA ${ }^{8,9}$. The MRPP1-MRPP2 subcomplex enhances the efficiency of mitochondrial tRNA $3^{\prime}$ processing and presents the nascent tRNA to the mitochondrial CCA tRNA nucleotidyltransferase to assist the maturation of mitochondrial tRNA ${ }^{10}$. Hence, HSD17B10 plays an important role in mitochondrial tRNA maturation.

HSD17B10, mapping at Xp11.2, is a highly conserved gene across a large evolutionary distance from nematodes to mammals, implying that HSD17B10 plays a crucial role $^{11}$. HSD17B10 missense mutations cause HSD10 mitochondrial disease (HSD10MD), with features including progressive neurodegeneration, psychomotor retardation,

\section{(c) The Author(s) 2020}

(c) (i) Open Access This article is licensed under a Creative Commons Attribution 4.0 International License, which permits use, sharing, adaptation, distribution and reproduction cc in any medium or format, as long as you give appropriate credit to the original author(s) and the source, provide a link to the Creative Commons license, and indicate if changes were made. The images or other third party material in this article are included in the article's Creative Commons license, unless indicated otherwise in a credit line to the material. If material is not included in the article's Creative Commons license and your intended use is not permitted by statutory regulation or exceeds the permitted use, you will need to obtain permission directly from the copyright holder. To view a copy of this license, visit http://creativecommons.org/licenses/by/4.0/. 
loss of mental and motor skills, seizures, cardiomyopathy, and vision and hearing impairment ${ }^{12}$. HSD17B10 with mutations identified from cases of HSD10MD show loss of function in dehydrogenase activity and mitochondrial tRNA maturation, resulting in mitochondrial dysfunction ${ }^{13-15}$. HSD17B10 has a special D-loop structure that interacts with amyloid-beta $(A \beta)^{16}$. In Alzheimer disease (AD), HSD17B10 activity is inhibited by interacting with intracellular $A \beta$ which may contribute to the neuronal dysfunction associated with $\mathrm{AD}^{17}$.

Post-translational modification of proteins is of great importance in regulating protein functions. A study showed that Parkin can regulate mitochondrial abundance of HSD17B10 in a ubiquitin-dependent manner to promote mitochondrial elongation ${ }^{18}$. In our previous study, we identified HSD17B10 from NAD-dependent deacetylase Sirtuin 3 (SIRT3) complexes ${ }^{19}$. In this study, we show that HSD17B10 is deacetylated by SIRT3, and its function is regulated by its acetylation levels. Our results suggest a post-translational modification pathway that regulates the functions of HSD17B10 and results in a change of cellular phenotype.

\section{Materials and methods}

\section{Protein purification}

Constructs were transfected into HEK293T cells by Polyethyleneimine (PEI). After $48 \mathrm{~h}$, cells were harvested and lysed in BC200 buffer $(200 \mathrm{mM} \mathrm{NaCl}, 20 \mathrm{mM}$ pH7.3 Tris, 20\% glycerol, 0.2\% NP-40). Cell lysates were incubated with anti-Flag $\mathrm{M}_{2}$ beads (Sigma, USA)/anti-HA beads (Roche, Switzerland) at $4{ }^{\circ} \mathrm{C}$ overnight. The beads were washed with $\mathrm{BC} 100$ four times and eluted with Flag peptide (Sigma)/HA peptide (Roche) at $4{ }^{\circ} \mathrm{C}$ for $4 \mathrm{~h}$.

GST and GST fusion proteins were expressed in Rosetta (DE3) (CWBiotech, Beijing, China) bacterial cells, treated with $0.8 \mathrm{M}$ IPTG (Sigma) at $37^{\circ} \mathrm{C}$ for $4 \mathrm{~h}$ to induce fusion protein expression. Bacterial cells were harvested and suspended in $10-20 \mathrm{~mL}$ PBS. The same volume of BC1000 ( $1 \mathrm{M} \mathrm{NaCl}, 20 \mathrm{mM}$ Tris, 40\% glycerol, $2 \%$ Triton $\mathrm{X}-100)$ was added and bacterial cells were lysed by sonication. The lysates were incubated with GST-agarose beads (Novagen) at $4{ }^{\circ} \mathrm{C}$ overnight. The beads were washed with $\mathrm{BC} 100$ four times and eluted with Glutathione (GSH) at $4{ }^{\circ} \mathrm{C}$ for $4 \mathrm{~h}$.

SDS-PAGE followed by Coomassie blue staining was used to quantify the amount of proteins purified from bacteria and/or cells. Bovine serum albumin (BSA) was diluted into concentration gradients, used as protein standards.

\section{Western blotting}

HEK293T cells, HCT116 cells or U2OS cells were harvested and lysed in BC100 buffer $(100 \mathrm{mM} \mathrm{NaCl}$, $20 \mathrm{mM}$ pH7.3 Tris, 20\% glycerol, 0.2\% NP-40). Cell lysates were incubated with anti-Flag $\mathrm{M}_{2}$ beads (Sigma, USA)/ anti-HA beads (Roche, Switzerland) at $4{ }^{\circ} \mathrm{C}$ overnight. For endogenous immunoprecipitation, cell lysates were incubated with $1 \mu \mathrm{g}$ anti-HSD17B10 (Abcam, UK), anti-SIRT3 (Cell Signaling Technology, USA), or normal mouse IgG, normal rabbit IgG (Santa Cruz Biotechnology, USA) at $4{ }^{\circ} \mathrm{C}$ overnight. Protein A/G agarose beads (Santa Cruz Biotechnology, USA) were added into the lysates and incubated at $4{ }^{\circ} \mathrm{C}$ for $8 \mathrm{~h}$. Beads were washed with BC100 four times and eluted by $0.1 \mathrm{M}$ glycine (Sigma, USA) at $4{ }^{\circ} \mathrm{C}$ for $10 \mathrm{~min}$ and neutralized with $1 \mathrm{M}$ Tris Base. The elution was subjected to western blot and immunoblotted with indicated antibodies.

All cell lines were bought from ATCC. The information of antibodies was as follows: Anti-Flag (Sigma-Aldrich, Cat\#F-3165), Anti-HA (Thermo Fisher Scientific, Cat\#26183), Anti-HSD17B10 (Abcam, Cat\#ab10260), Anti-SIRT3 (Cell Signaling Technology, Cat\#2627 S), Anti-CBP (Cell Signaling Technology, Cat\#7389 S), Antipan acetyllysine (Cell Signaling Technology, Cat\#9441 L), Anti- $\beta$-actin (Santa Cruz Biotechnology, Cat\#sc-47778), Anti-DO-1 (Cell Signaling Technology, Cat\#18032 S), Anti-p62 (Proteintech, Cat\#18420-1-AP), and Anti-LC3B (Sigma-Aldrich, Cat\#L7543).

\section{GST pull-down and in vitro acetylation assay}

Flag-tagged HSD17B10 and SIRT3 were purified from HEK293T cells. GST and GST fusion proteins were purified from Rosetta (DE3) bacterial cells and bound to GSTagarose beads. Flag-tagged proteins and GST fusion proteins were incubated in buffer $\mathrm{BC} 200$ at $4{ }^{\circ} \mathrm{C}$ overnight. Beads were washed with $\mathrm{BC} 100$ four times and boiled in the SDS sample buffer. The elution was subjected to western blot and immunoblotted with indicated antibodies.

For in vitro acetylation assay, the GST fusion proteins were purified from Rosetta (DE3) bacterial cells. The reaction mixture contained $10 \times$ Ac Buffer $1(200 \mathrm{mM}$ pH8.0 HEPES, $10 \mathrm{mM}$ DTT, $10 \mathrm{mM}$ PMSF, $1 \mathrm{mg} / \mathrm{ml} \mathrm{BSA}$ ), acetyl-CoA $(17 \mathrm{ng} / \mu \mathrm{l}), \mathrm{CBP}$ and GST fusion proteins. The reaction mixture was incubated at $37^{\circ} \mathrm{C}$ for $2 \mathrm{~h}$ and boiled in the SDS sample buffer.

\section{In vitro deacetylation assay}

Flag-tagged HSD17B10 and SIRT3 were purified from HEK293T cells. The reaction mixture contained $10 \times \mathrm{Ac}$ Buffer1, $2 \times$ DeAc Buffer2 (8 mM MgCl $2,100 \mathrm{mM} \mathrm{NaCl}$, $20 \%$ glycerol), and acetylated HSD17B10. SIRT3 and $\mathrm{NAD}^{+}(1 \mathrm{mM})$ were present or absent as needed. The reaction mixture was incubated at $37^{\circ} \mathrm{C}$ for $2 \mathrm{~h}$ and boiled in the SDS sample buffer.

\section{HSD17B10 enzymatic activity assays}

HSD17B10 dehydrogenase activity is measured in the reverse direction for the reduction of acetoacetyl-CoA to L-3-hydroxyacyl-CoA accompanied by the oxidation of $\mathrm{NADH}$ to $\mathrm{NAD}^{+20}$. The assay mixture contains $0.1 \mathrm{M}$ 
pH7.0 potassium phosphate, $0.2 \mathrm{mg} / \mathrm{ml}$ bovine serum albumin, $0.2 \mathrm{mM} \mathrm{NADH}$, and various concentrations of acetoacetyl-CoA $(0-300 \mu \mathrm{M})$. The assay was started by the addition of $50 \mathrm{nM}$ HSD17B10. The decrease in absorbance at $340 \mathrm{~nm}$ was measured. NADH standard curve was established to measure substrate conversion. Kinetic parameters were calculated from initial-velocity by direct curve fitting using non-linear regression analysis, and Michaelis-Menten plots were generated via GraphPad Prism 7.0.

\section{Generation of stable HSD17B10/SIRT3 CRISPR-Cas9 cell lines}

Single guide RNA (sgRNA) sequences were as follows: SIRT3: 5'-CACCGCTCTACACGCAGAACATCGA-3'; HSD17B10 1\#: 5'-CACCGCCACGGCGGAGCGA CTT GT-3';

HSD17B10 5\#: 5'-CACCGCATGCCCACTATTCCCC CCT-3'

sgRNAs were cloned into LentiCRISPR-V2 vector, and the plasmids were transfected with packaging plasmids pSPAX2 and pMD.2 G (at 4:3:1 ratio) into HEK293T cells. The medium was changed after $8-10 \mathrm{~h}$. Viruses were collected at $48 \mathrm{~h}$ post-transfection to infect target cells $\left(\mathrm{U}_{2} \mathrm{OS}\right.$ or HCT116). The infected cells were selected for stable cell lines with $1 \mu \mathrm{g} / \mathrm{ml}$ puromycin for 2 weeks.

\section{Knockdown of CBP}

Small interfering RNA (siRNA) sequences were as follows: 1\#: CGGCACAGCCUCUCAGUCA(dTdT)

2\#: GAGGUCGCGUUUACAUAAA(dTdT)

siRNAs were transfected into HEK293T cells by RNAiMAX (ThermoFisher, USA). The medium was changed after $24 \mathrm{~h}$. Cells were harvested at $48 \mathrm{~h}$ posttransfection and lysed in BC100 buffer $(100 \mathrm{mM} \mathrm{NaCl}$, $20 \mathrm{mM}$ pH7.3 Tris, $20 \%$ glycerol, $0.2 \%$ NP-40). Cell lysates were incubated with anti-acetyllysine agarose (Immunechem, Canada) at $4{ }^{\circ} \mathrm{C}$ overnight. Beads were washed with $\mathrm{BC} 100$ four times and eluted by $0.1 \mathrm{M}$ glycine (Sigma, USA) at $4{ }^{\circ} \mathrm{C}$ for $10 \mathrm{~min}$ and neutralized with $1 \mathrm{M}$ Tris Base. The elution was subjected to western blot and immunoblotted with indicated antibodies.

\section{Measurement of cellular $\mathrm{NAD}^{+} / \mathrm{NADH}$ levels}

$\mathrm{NAD}^{+} / \mathrm{NADH}$ levels in HCT116 cells were measured with the NAD/NADH Quantification Kit (Sigma, USA). $2 \times 10^{5}$ cells were collected for each assay. Cells were extracted and samples were deproteinized before use in assay. Samples were divided into two parts for $\mathrm{NAD}_{\text {total }}$ and $\mathrm{NADH}$ only measurement. The absorbance at $450 \mathrm{~nm}\left(A_{450}\right)$ was measured. All the samples were run in triplicate. $\mathrm{NAD}^{+} / \mathrm{NADH}=\left(\mathrm{NAD}_{\text {total }}-\mathrm{NADH}\right) /$ $\mathrm{NADH}$.

\section{RT-qPCR}

Total RNA was extracted using Trizol reagent (Sigma, USA). $2 \mu \mathrm{g}$ RNA was reverse-transcribed into cDNA using the Revertra ace qPCR RT Kit (TOYOBO, Japan). Realtime PCR was performed in triplicate using AceQ qPCR SYBR green master mix (Vazyme, Nanjing, China) by the ABI 7500/7500 fast Real-Time PCR systems (Applied Biosystems, ThermoFisher, USA). The amount of tRNA was calculated by $\triangle \triangle \mathrm{Ct}$ method. The human 18S RNA was used as the internal control. For pre-tRNAs, primers were designed to span the cleavage sites. For mature tRNAs, primers were designed within gene exon, and therefore amplified both pre- and mature mitochondrial tRNAs. The primers were as follows:

mt pre-tRNA ${ }^{\text {Pro }}$ : 5'-GATGGGTGAGTCAATACTTGG G- $3^{\prime}$ and $5^{\prime}$-CCATTAGCACCCAAAGCTAAGA- $3^{\prime}$; $\mathrm{mt}$ pre-tRNA $^{\text {Val }}$ : 5'-AGAGGAGACAAGTCGTAACATGG-3' and $5^{\prime}$-GGTGCTTTGTGTTAAGCTACACTCTGG-3'; mt pre-tRNA ${ }^{\text {Ile }}: 5^{\prime}$-GCGAATTCAATCTCCAGCA-3' and 5'-G AAATAAGGGGGTTTAAGC-3'; mt tRNA ${ }^{\text {Pro }}$ : $5^{\prime}$-TCAG AGAAAAAGTCTTTAACTCCACC- $3^{\prime}$ and $5^{\prime}$-ATAGTTT AAATTAGAATCTTAGCTTTGGGT-3'; mt tRNA ${ }^{\mathrm{Val}}: 5^{\prime}-\mathrm{G}$ TGTAGCTTAACACAAAGCACCC- $3^{\prime}$ and $5^{\prime}$-TCAGAG CGGTCAAGTTAAGTTG-3'; mt tRNA ${ }^{\text {Ile }}$ : 5'-TCTTTAT ACAGACTATTTTC- $3^{\prime}$ and $5^{\prime}$-TAGAAATAAGGGGG TTTAAG-3'; $18 \mathrm{~S}$ RNA: $5^{\prime}$-GCCGCTAGAGGTGAAA TTCTT-3' ${ }^{\prime}$ and $5^{\prime}$-CTTTCGCTCTGGTCCGTCTT-3'.

\section{Mitochondrial membrane potential}

HCT116 cells were grown in glass bottom cell culture dish ( $\$ 20 \mathrm{~mm}$, Nest, China) to $70 \%$ confluent. $200 \mathrm{nM}$ Mito Tracker Green (Invitrogen, USA) and $10 \mathrm{nM}$ TMRM (ThermoFisher, USA) were incubated with cells for $30 \mathrm{~min}$, followed by washing twice with HBSS media. Cells were imaged live by confocal microscopy and the intensity of fluorescence (ex: $490 \mathrm{~nm}$, em: $516 \mathrm{~nm}$ for Mito Tracker Green; ex: $490 \mathrm{~nm}$, em: $550 \mathrm{~nm}$ for TMRM) was recorded to determine the uncollapsed proton gradient to measure mitochondrial membrane potential.

\section{Cell growth}

HCT116 cells were seeded into six-well plates and were counted every $24 \mathrm{~h}$ to establish cell growth curves. HCT116 cells were seeded into $6 \mathrm{~cm}$ dishes. After culturing for 7 days, cells were washed with PBS three times, fixed with $4 \%$ paraformaldehyde, and stained with $0.2 \%$ crystal violet. Pictures were shot to show colony formation. HCT116 cells were seeded into 96-well plates and cultured for $48 \mathrm{~h} .10 \mu \mathrm{l} \mathrm{CCK}-8$ was added into $100 \mu \mathrm{l}$ DMEM (no phenol red, Gibco, USA) in each well. The plate was incubated for $1 \mathrm{~h}$ in the incubator $\left(37^{\circ} \mathrm{C}, 5 \%\right.$ $\left.\mathrm{CO}_{2}\right)$. The absorbance at $450 \mathrm{~nm}\left(A_{450}\right)$ was finally measured using microplate reader. 


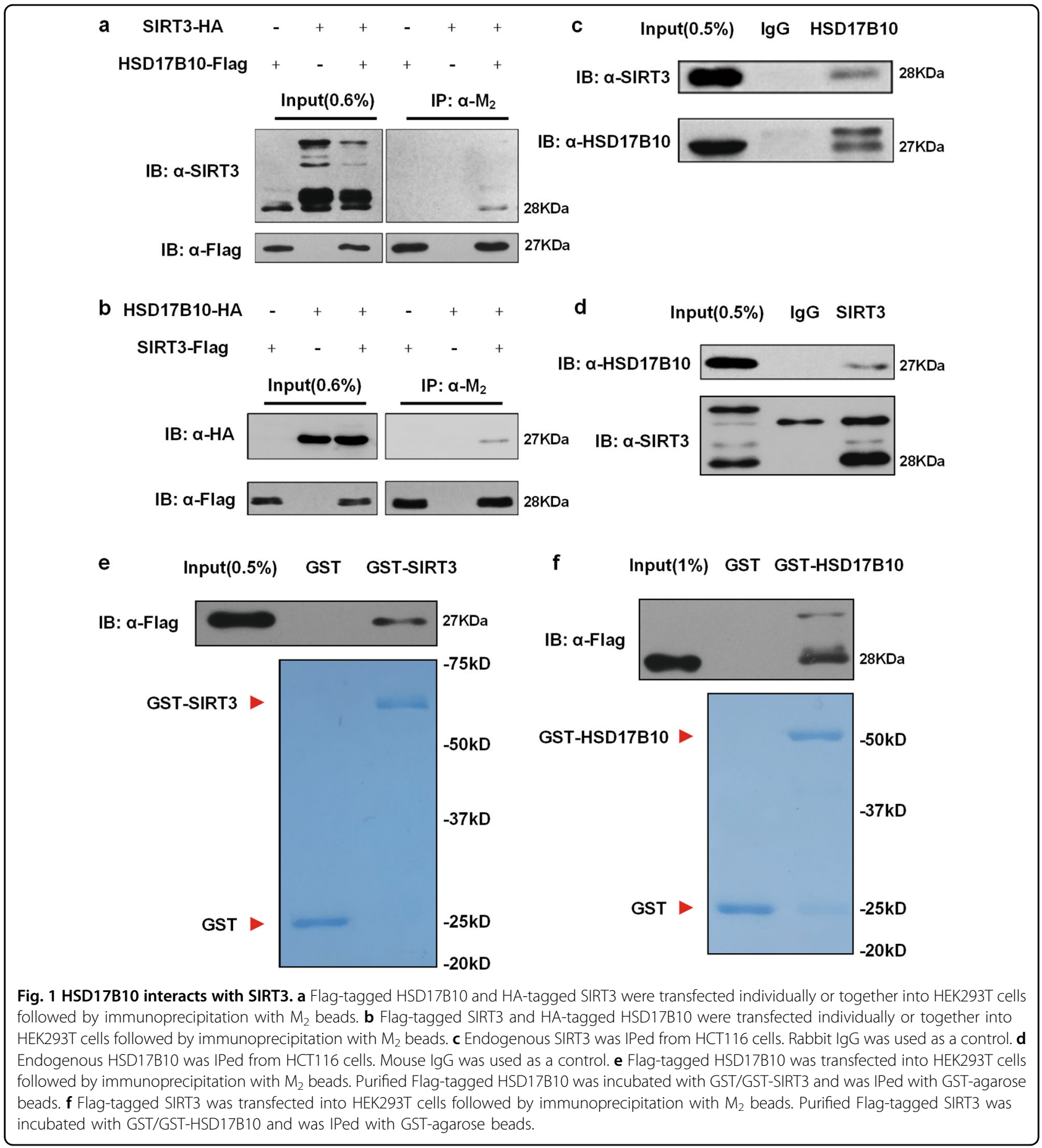

\section{Statistical analysis}

Experiments in Figs. 1 and 2e, f were performed twice. The other experiments were performed three times. The repeated experiments showed similar results. Sample sizes were regularly chosen as three replicates. Statistical analysis was performed via Graphpad prism 7.0. Data were expressed as mean \pm standard deviation (S.D.) and S.D. was calculated to estimate the variation within each group of data.

\section{Results}

HSD17B10 interacts with SIRT3

In our previous report, we purified SIRT3 complex by immunoprecipitation and mass spectrometry. HSD17B10 was found in the complex ${ }^{19}$. To verify the interaction between HSD17B10 and SIRT3, we overexpressed tagged SIRT3 and HSD17B10 in HEK293T cells and performed co-immunoprecipitation assays. SIRT3 can interact with 


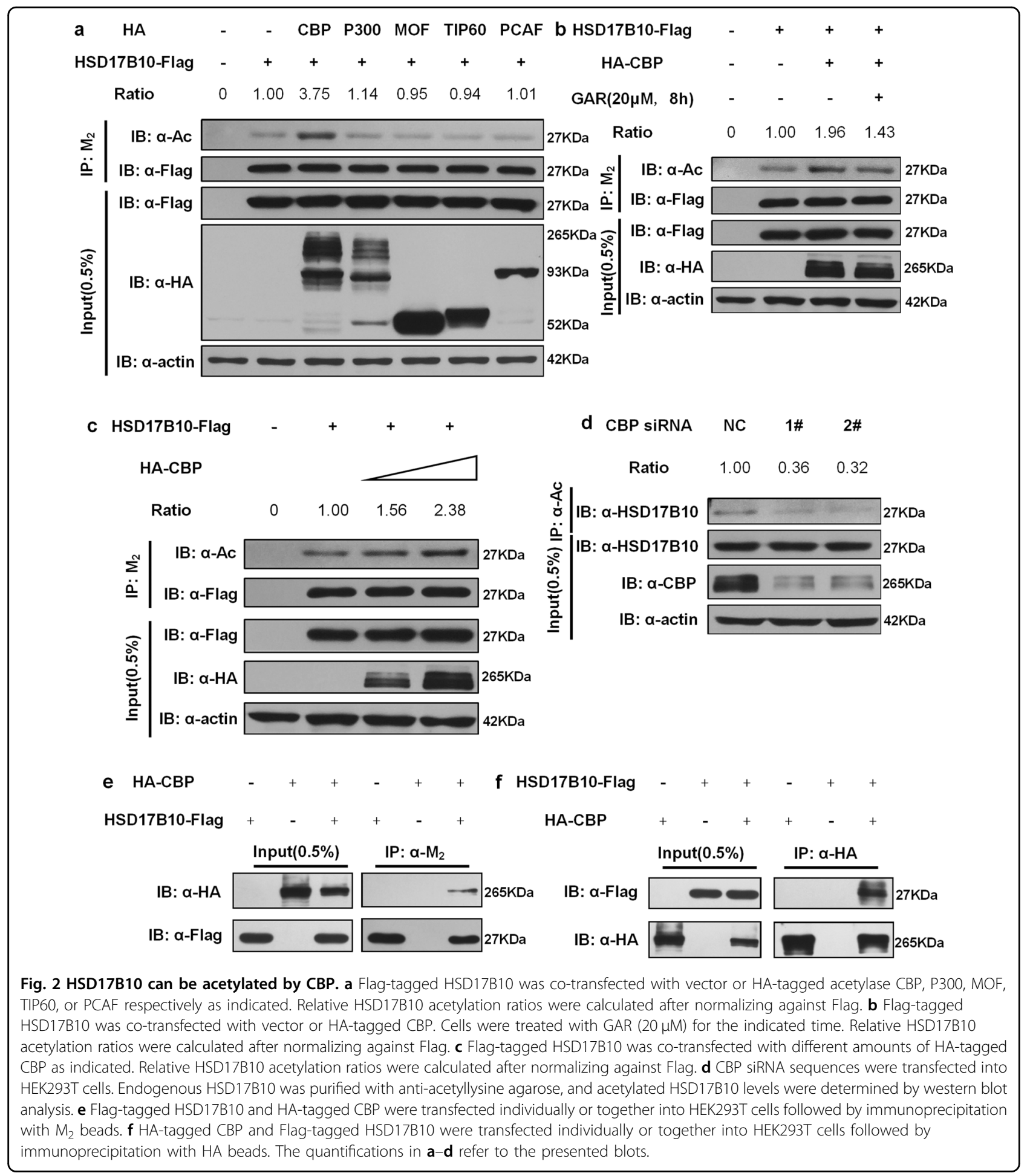

HSD17B10 (Fig. 1a), and vice versa (Fig. 1b). SIRT3HSD17B10 interaction was further confirmed by the coimmunoprecipitation of endogenous SIRT3 and HSD17B10 (Fig. 1c, d). We also performed GST pulldown assay to determine if HSD17B10 and SIRT3 can directly interact with each other. The results showed that GST-SIRT3 pulled down HSD17B10 (Fig. 1e) and GSTHSD17B10 pulled down SIRT3 (Fig. 1f). Taken together, we demonstrated that HSD17B10 directly interacts with SIRT3 both in vivo and in vitro. 


\section{HSD17B10 is acetylated by CBP}

The interaction between HSD17B10 and SIRT3 prompted us to consider whether HSD17B10 is a novel target of SIRT3. Therefore, we first examined whether HSD17B10 could be acetylated. We co-transfected Flag-HSD17B10 with different acetyltransferases in HEK293T cells and incubated the cell lysates with $\mathrm{M}_{2}$ beads to immunoprecipitate HSD17B10. HSD17B10 acetylation was detected when co-transfected with CBP or p300, and CBP gave a stronger acetylation signal (Fig. 2a). Hence, CBP is the major acetyltransferase for HSD17B10. Garcinol (GAR) is a common CBP acetylation activity inhibitor. Treating cells with GAR for the indicated time inhibited HSD17B10 acetylation by CBP (Fig. 2b). Flag-tagged HSD17B10 cotransfected with different amounts of HA-tagged CBP showed that HSD17B10 acetylation increased as the amount of CBP increased (Fig. 2c). siRNA knockdown of CBP in HEK293T cells decreased acetylation levels of HSD17B10 (Fig. 2d). Flag-tagged HSD17B10 and HAtagged CBP transfected individually or together into HEK293T cells followed by immunoprecipitation showed that HSD17B10 and CBP could interact with each other (Fig. 2e, f). In conclusion, we demonstrated that HSD17B10 can be acetylated and CBP is the major acetyltransferase.

\section{HSD17B10 is deacetylated by SIRT3}

We further explored if SIRT3 can deacetylate HSD17B10. We transfected Flag-tagged HSD17B10 into HEK293T cells and treated cells with nicotinamide (NAM) and Trichostatin A (TSA) individually or simultaneously for the indicated time. We found that HSD17B10 acetylation levels were higher after treating cells with NAM, a specific inhibitor of the sirtuins, whereas treating with TSA, an inhibitor of other HDACs, did not affect HSD17B10 acetylation (Fig. 3a). Meanwhile, treating with NAM together with TSA did not further enhance HSD17B10 acetylation compared to treating with NAM alone (Fig. 3a). These results indicate that HSD17B10 acetylation is regulated by the sirtuins family.

Study on HSD17B10 subcellular location shows that it is distributed in mitochondria ${ }^{2}$. SIRT3, SIRT4, and SIRT5 are three mitochondrial members of the sirtuins family. Thus, we co-transfected Flag-tagged HSD17B10 together with HA-tagged SIRT3, SIRT4, or SIRT5 into HEK293T cells. Compared to SIRT4 or SIRT5, SIRT3 gave the strongest deacetylation activity of HSD17B10 (Fig. 3b). Knockout of SIRT3 in U2OS cells showed increased acetylation levels of HSD17B10 (Fig. 3c), indicating that HSD17B10 is hyperacetylated in SIRT3 knockout cells. Flag-tagged HSD17B10 co-transfected with different amounts of HA-tagged SIRT3 showed that HSD17B10 acetylation decreased as the amount of SIRT3 increased (Fig. 3d). We found that SIRT3 H248Y, a SIRT3 catalytic center mutant, cannot deacetylate
HSD17B10 as much as SIRT3 WT (Fig. 3e). In addition, we conducted an in vitro deacetylation assay and found that SIRT3 can deacetylate HSD17B10 in vitro and the deacetylation process is $\mathrm{NAD}^{+}$-dependent (Fig. 3f). Thus, we concluded that SIRT3 can deacetylate HSD17B10 both in vivo and in vitro.

\section{Identify the major acetylation sites of HSD17B10}

To identify the major acetylation sites of HSD17B10, we purified acetylated HSD17B10 for mass spectrometry (MS) (Fig. 4a). The result showed that K52, K53, K99, K105 and K172 are putative acetylation sites. We further conducted an in vitro acetylation assay. HSD17B10 was purposely separated into three fractions as indicated in Fig. 4b. HSD17B10 GST fusion protein N-terminus (NT), middle (M) and full-length (FL) showed acetylation signal but not C-terminus (CT), indicating that acetylation sites of HSD17B10 are at NT and M fractions, which also excluded CT located lysine residues K172 and K212. HSD17B10 is a highly evolutionary conservation protein ${ }^{11}$, and conserved lysine residue usually plays a critical role in protein acetylation and can affect protein functions. We analyzed HSD17B10 amino acid sequence conservation and identified K9, K79, K81, K99, K104 and K105 as highly conserved lysines (Fig. 4c). We constructed KR mutant plasmid which is a mimic for deacetylation status, and transfected WT and KR mutant HSD17B10 into HEK293T cells to examine their acetylation status. We found that K9R, K52R, K53R, K79R, K99R and K105R decreased HSD17B10 acetylation levels (Fig. 4d). Altogether, these data showed that K9, K52, K53, K79, K99 and K105 are putative acetylation lysine residues of HSD17B10.

\section{HSD17B10 acetylation affects its enzymatic activity and the formation of mt-tRNA splicing complex}

HSD17B10 contains mitochondrial dehydrogenase activity $^{1}$, and acts as a component of mt-tRNA splicing complex mitochondrial ribonuclease $\mathrm{P}^{6}$. We investigated if HSD17B10 acetylation regulates these functions. We purified deacetylated and hyperacetylated HSD17B10 to carry out an in vitro enzymatic activity assay ${ }^{20}$. Kinetic parameters were calculated from initial-velocity by direct curve fitting using non-linear regression analysis. Michaelis-Menten plots and kinetic parameters were shown in Fig. S1. $k_{\text {cat }} / K_{\mathrm{M}}$ was calculated to represent catalytic efficiency. Acetylation of HSD17B10 significantly reduced its enzymatic activity compared to deacetylated HSD17B10 (Fig. 5a). We then purified HSD17B10 WT and KR mutants which are putative acetylated lysine residues of HSD17B10 (Fig. 4d) for the in vitro enzymatic activity assay. Interestingly, we found that only K79R, K99R and K105R dramatically increased HSD17B10 enzymatic activity (Fig. 5b). Meanwhile, in terms of interaction with TRMT10C, a component of mitochondrial ribonuclease P, 


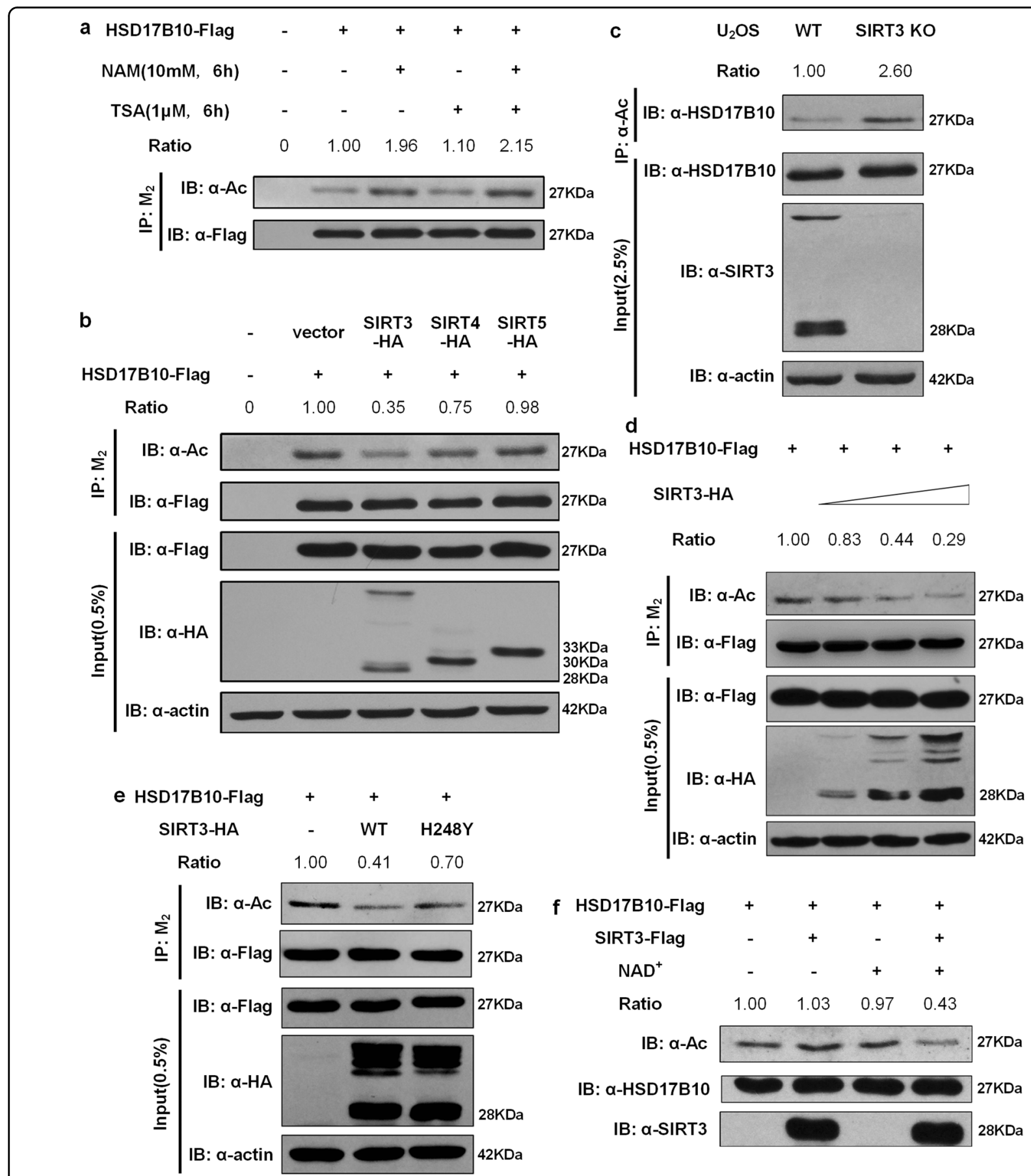

Fig. 3 SIRT3 is the main deacetylase for HSD17B10. a Flag-tagged HSD17B10 was transfected into HEK293T cells, and cells were treated with $10 \mathrm{mM}$ NAM and/or $1 \mu \mathrm{M}$ TSA for the indicated time. Relative HSD17B10 acetylation ratios were calculated after normalizing against Flag. $\mathbf{b}$ Flagtagged HSD17B10 was co-transfected with vector or HA-tagged SIRT3/SIRT4/SIRT5 respectively as indicated. Relative HSD17B10 acetylation ratios were calculated after normalizing against Flag. c Endogenous HSD17B10 was purified with anti-acetyllysine agarose in WT or SIRT3 knockout U2OS cells. Acetylated HSD17B10 levels were determined by western blot analysis. d Flag-tagged HSD17B10 was co-transfected with different amounts of HA-tagged SIRT3 as indicated. Relative HSD17B10 acetylation ratios were calculated after normalizing against Flag. e Flag-tagged HSD17B10 was COtransfected with vector, HA-tagged WT SIRT3 or the catalytic center mutation SIRT3 H248Y respectively. Relative HSD17B10 acetylation ratios were calculated after normalizing against Flag. f Flag-tagged HSD17B10 and SIRT3 were respectively transfected into HEK293T cells and were purified with $\mathrm{M}_{2}$ beads. In vitro deacetylation assay was performed as indicated. Relative HSD17B10 acetylation ratios were calculated after normalizing against Flag. The quantifications in this figure refer to the presented blots. 


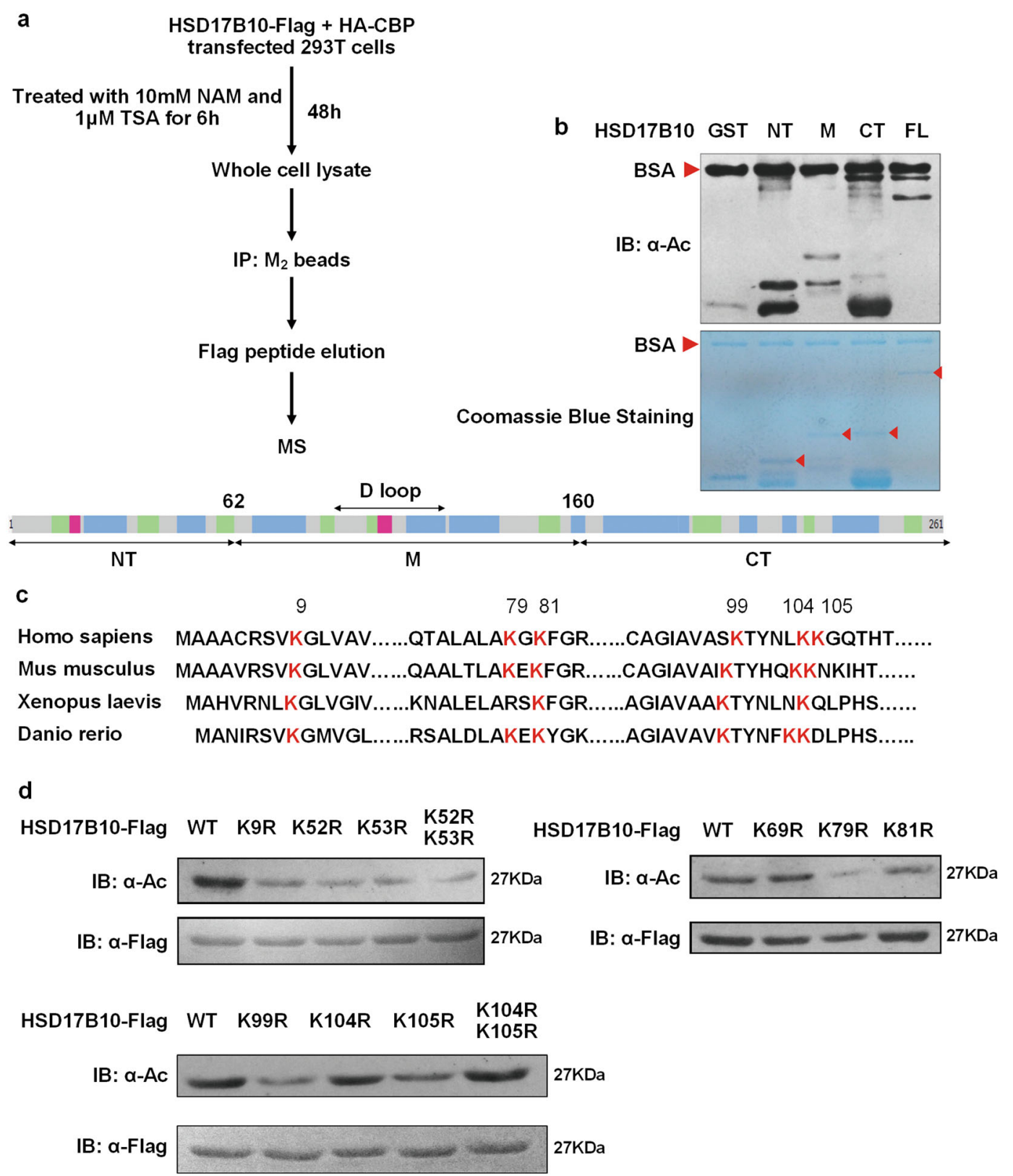

Fig. 4 Identify the main acetylation sites of HSD17B10. a Flag-tagged HSD17B10 was co-transfected with HA-tagged CBP, and cells were treated with $10 \mathrm{mM}$ NAM for $6 \mathrm{~h}$. Whole-cell lysate was collected, IPed with $\mathrm{M}_{2}$ beads and eluted by Flag peptide. After SDS-PAGE gel separating and Coomassie blue staining, the specific band for HSD17B10 was analyzed by mass spectrometry. b HSD17B10 GST N-terminal (NT), middle (M), C-terminus (CT) and full-length (FL) plasmids were constructed, and in vitro acetylation assay was conducted. GST protein was used as a negative control. c HSD17B10 amino acid sequence conservation was analyzed via sequence analyzing software MEGA. d HSD17B10 KR mutant (mimicking the deacetylation status) plasmids were constructed. Flag-tagged HSD17B10 WT and KR mutants were individually transfected and cells were treated with $10 \mathrm{mM}$ NAM for $6 \mathrm{~h}$. Relative HSD17B10 acetylation ratios were calculated after normalizing against Flag.

HSD17B10 WT and KR mutants showed similar results. K79R, K99R and K105R mutants promoted interaction between TRMT10C and HSD17B10 more strikingly (Fig. 5c).

Thus, we concluded that K79R, K99R and K105R are three major functional acetylation lysine residues and constructed multiple lysine mutant plasmids $3 K R$ and 3KQ (mimic for hyperacetylation status) for further investigation. We transfected WT and 3KR mutant HSD17B10 into HEK293T cells treated with SIRT3 inhibitor NAM, and $3 K R$ acetylation levels were much reduced (Fig. $5 \mathrm{~d}$ ). We then purified WT, 3KR and $3 \mathrm{KQ}$ 

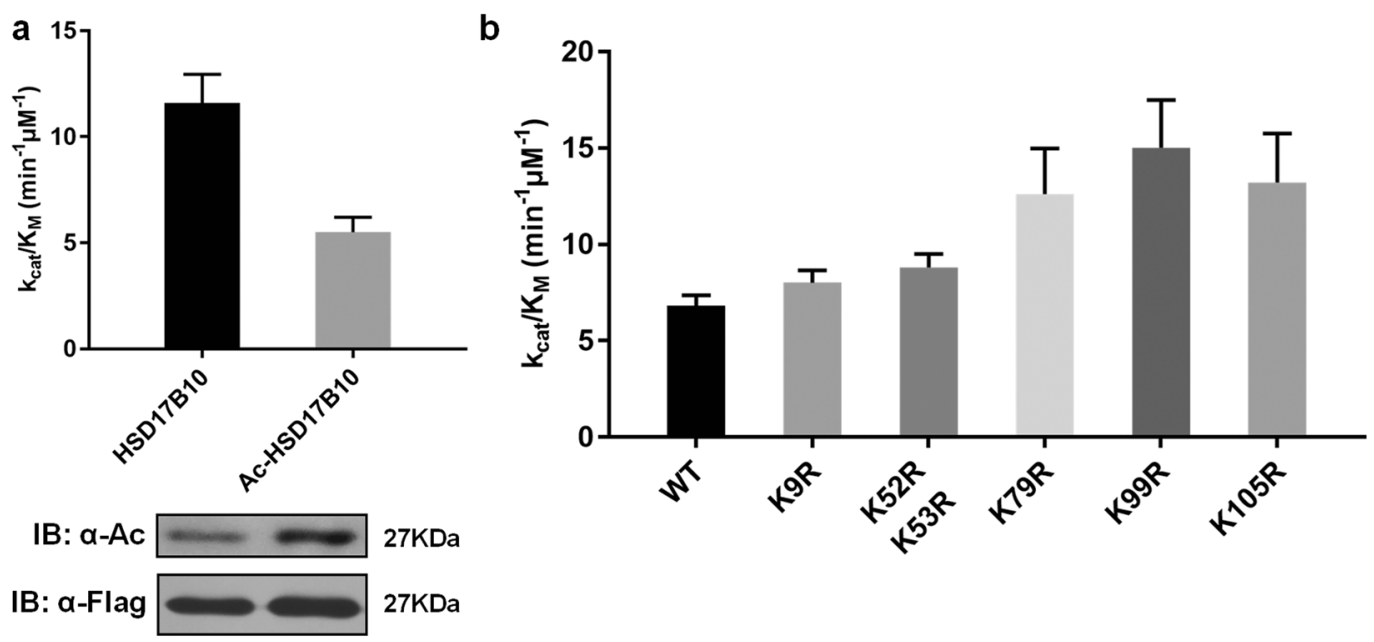

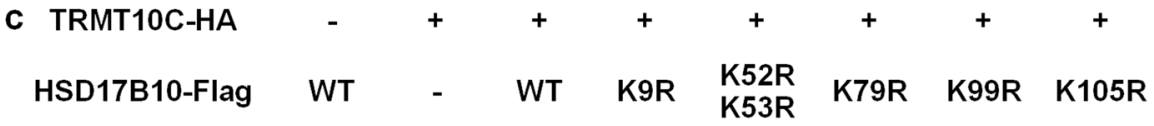
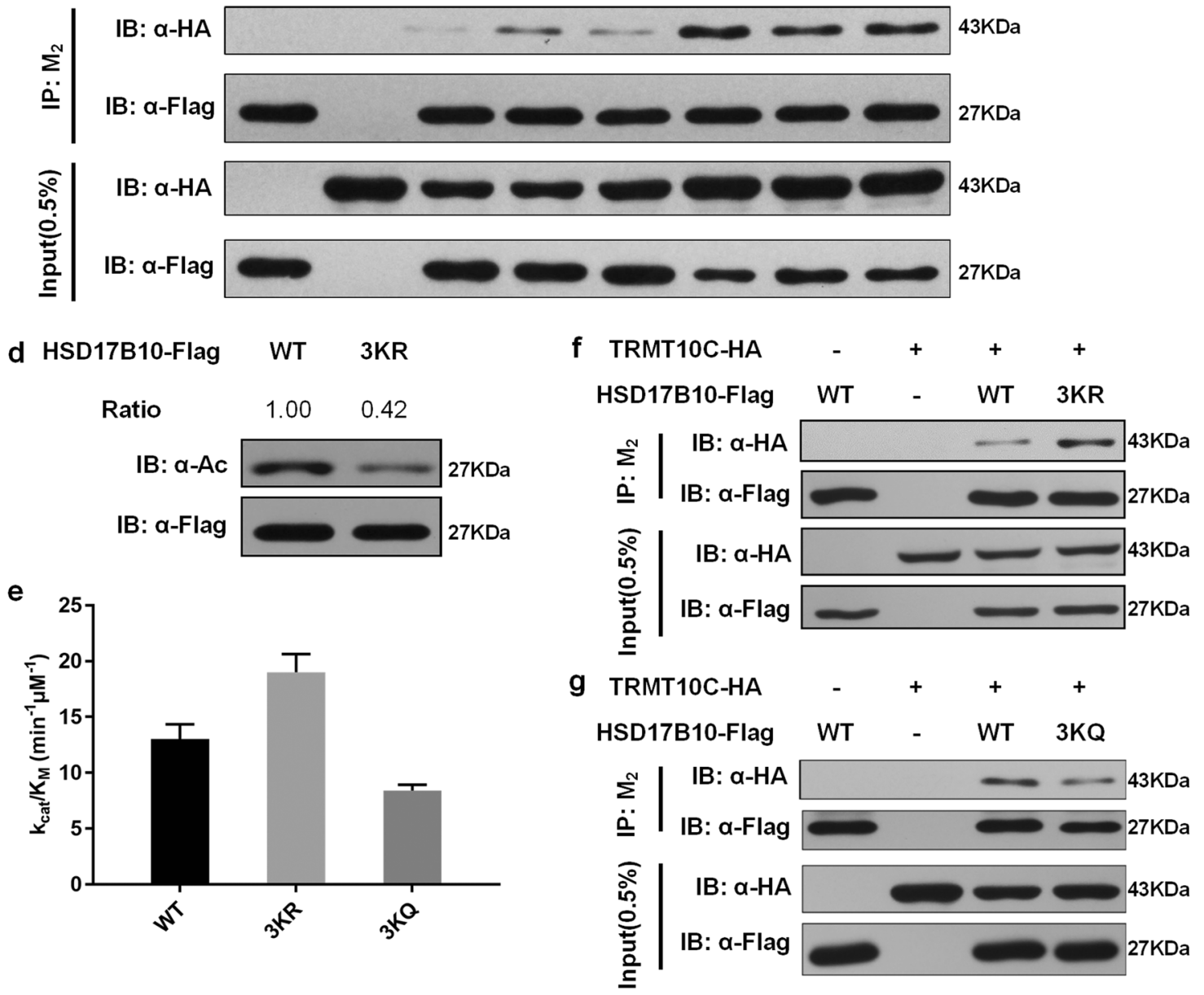

Fig. 5 (See legend on next page.) 
(see figure on previous page)

Fig. 5 HSD17B10 acetylation affects its enzymatic activity and the formation of mt-tRNA splicing complex. a Flag-tagged HSD17B10 was transfected into HEK293T cells, and cells were treated with or without $10 \mathrm{mM}$ NAM for $6 \mathrm{~h}$ for deacetylated and hyperacetylated HSD17B10 purification. The L-3-hydroxyacyl-CoA dehydrogenase activity assay of HSD17B10 was conducted with acetoacetyl-CoA as the substrate and NADH as the cofactor, and changes of the absorbance at $340 \mathrm{~nm}$ were measured to show the decrease of $\mathrm{NADH} . \mathrm{K}_{\mathrm{cat}} / K_{\mathrm{M}}$ was calculated to represent catalytic efficiency. Data were shown as mean \pm S.D. $(n=3)$. b Flag-tagged HSD17B10 WT and KR mutants were individually transfected, and cells were treated with $10 \mathrm{mM}$ NAM for $6 \mathrm{~h}$. The L-3-hydroxyacyl-CoA dehydrogenase activity of HSD17B10 was measured as previously described. Data were shown as mean \pm S.D. $(n=3)$. c Flag-tagged HSD17B10 WT and KR mutants were co-transfected with HA-tagged TRMT10C into HEK293T cells followed by immunoprecipitation with $\mathrm{M}_{2}$ beads. $\mathbf{d}$ Flag-tagged HSD17B10 WT and 3KR were transfected into HEK293T cells, and cells were treated with $10 \mathrm{mM}$ NAM for $6 \mathrm{~h}$. Relative HSD17B10 acetylation ratios were calculated after normalizing against Flag. e Flag-tagged HSD17B10 WT, 3KR and $3 \mathrm{KQ}$ were individually transfected. The L-3-hydroxyacyl-CoA dehydrogenase activity of HSD17B10 was measured as previously described. Data were shown as mean \pm S.D. $(n=3)$. f Flag-tagged HSD17B10 WT and 3KR were co-transfected with HA-tagged TRMT10C into HEK293T cells followed by immunoprecipitation with $\mathrm{M}_{2}$ beads. g Flag-tagged HSD17B10 WT and 3KQ were co-transfected with HA-tagged TRMT10C into HEK293T cells followed by immunoprecipitation with $\mathrm{M}_{2}$ beads.

HSD17B10 for in vitro enzymatic activity assay, and HSD17B10 3KR mutant significantly enhanced its enzymatic activity compared to WT while $3 \mathrm{KQ}$ had the opposite effect (Fig. 5e). Co-IP assay showed the same result that $3 \mathrm{KR}$ mutant promoted interaction between TRMT10C and HSD17B10 whereas 3KQ acted inversely (Fig. 5f, g). Taken together, the above data demonstrated that K79, K99 and K105 are three major functional acetylation lysine residues of HSD17B10, and HSD17B10 acetylation affects its enzymatic activity and the formation of mt-tRNA splicing complex.

HSD17B10 acetylation regulates the intracellular functions

To further investigate the physiological functions of HSD17B10 acetylation, we first established HSD17B10 CRISPR-Cas9 knockout HCT116 cells. Knocking out HSD17B10 in mice is embryonic lethal ${ }^{21}$, and we found that knockout of HSD17B10 in cells also caused cell death. Therefore, we only obtained HSD17B10 knockdown clones (Fig. S2a). HSD17B10 functions as a mitochondrial dehydrogenase that catalyzes the beta-oxidation of androgens, estrogens and fatty acids ${ }^{3-5}$. As a result, $\mathrm{NAD}^{+}$will be changed to NADH. Consistent with this, our results showed that the $\mathrm{NAD}^{+} / \mathrm{NADH}$ ratio increased in HSD17B10 knockdown cells (Fig. S2b). Since knocking down HSD17B10 impaired the formation of mt-tRNA splicing complex, we chose $m t$ pre-tRNA ${ }^{\text {Pro }}, m t$ pre-tRNA ${ }^{\mathrm{Val}}$, and $m t$ pre-tRNA ${ }^{\text {Ile }}$ as three representative substrates. The amounts of these pretRNAs increased, as expected (Fig.S2c). Cells of HSD17B10deficient patients exhibit the loss of function in dehydrogenase activity and mitochondrial tRNA maturation, resulting in mitochondrial dysfunction ${ }^{13-15}$. Additionally, HSD17B10 has been proved to play a protective role for mitochondrial function ${ }^{18,21,22}$. Therefore, we used tetramethylrhodamine methyl ester (TMRM) staining intensity as an indicator for mitochondrial membrane potential. TMRM is a cell-permeant, cationic, red-orange fluorescent dye that is readily sequestered by active mitochondria, and in dysfunctional mitochondria TMRM is released into cytoplasm and the signal will disappear. We observed that, in HSD17B10 knockdown cells, TMRM staining intensity decreased, indicating that mitochondrial function was impaired (Fig. S2d).

We then re-expressed empty vector, HSD17B10 WT, $3 \mathrm{KR}$ and 3KQ in HSD17B10 knockdown HCT116 cells (Fig. 6a) to investigate whether HSD17B10 acetylation affected its intracellular functions. To determine the effect of HSD17B10 enzymatic activity on cellular $\mathrm{NAD}^{+}$/ NADH ratio, HSD17B10 K172A was served as an enzymatic negative control ${ }^{8}$. In Fig. 5, we demonstrated that HSD17B10 acetylation affects its enzymatic activity and the formation of mt-tRNA splicing complex. As expected, re-expressing WT HSD17B10 decreased $\mathrm{NAD}^{+} / \mathrm{NADH}$ ratio compared to vector, and $3 \mathrm{KR}$ mutant further reduced the ratio, while $3 \mathrm{KQ}$ barely changed the ratio compared to WT (Fig. 6b). Also, as shown in Fig. 6c, the amounts of $m t$ pre-tRNA ${ }^{\text {Pro }}$, $m t$ pre-tRNA ${ }^{\mathrm{Val}}$ and $\mathrm{mt}$ pretRNA $^{\text {Ile }}$ decreased with the re-expression of HSD17B10 WT and $3 K R$, while $3 K Q$ only slightly changed their amounts compared to empty vector. Consistently, mitochondrial function was rescued by HSD17B10 WT and $3 \mathrm{KR}$, while $3 \mathrm{KQ}$ had less effect (Fig. 6d). Taken together, we demonstrated that HSD17B10 acetylation regulated the mitochondrial function.

\section{HSD17B10 acetylation affects cell growth and cell resistance under stresses}

HSD17B10 expression level has been proved to impact cell growth $^{22}$. We established cell growth curve, CCK-8 and colony formation assays to verify that HSD17B10 knockdown HCT116 cells indeed had a slower proliferation rate (Fig. S3a-c). Re-expressing empty vector, HSD17B10 WT, 3KR and 3KQ in HSD17B10 knockdown HCT116 cells, we found that re-expression of WT HSD17B10 promoted cell growth and $3 K R$ enhanced this effect compared to vector, while cells re-expressed with $3 \mathrm{KQ}$ had a slower cell growth rate compared to cells re-expressed with WT (Fig. 7a, b, c).

HSD17B10 deficiency may induce cell apoptosis and overexpression of HSD17B10 can promote cell resistance to 


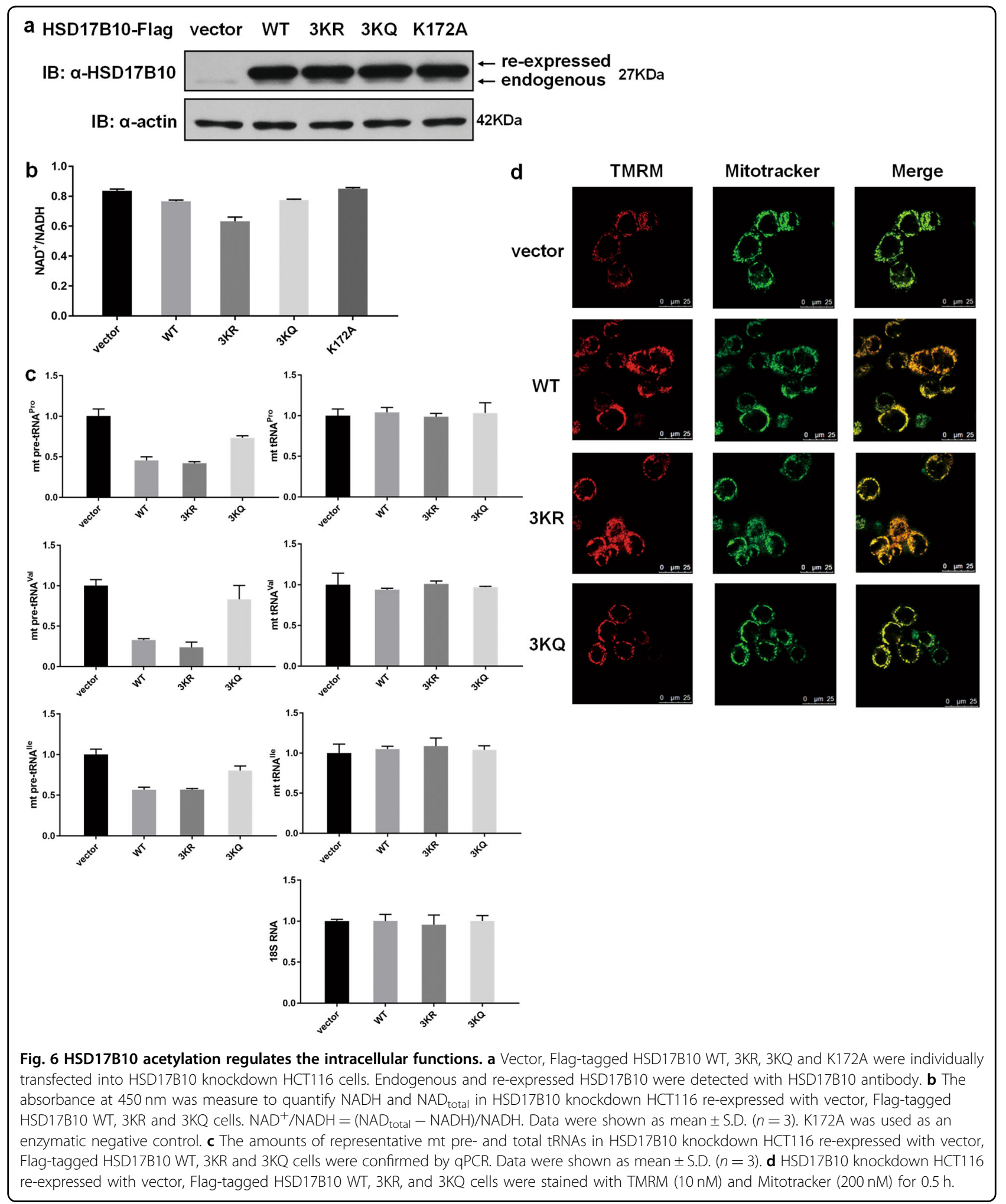

stress $^{13,22}$. SIRT3, the deacetylase of HSD17B10, is a stressresponsive factor as a deacetylase $\mathrm{e}^{23-25}$. To find if HSD17B10 acetylation regulates cell resistance to stress and whether SIRT3 participates in the process, we first treated cells with
$\mathrm{H}_{2} \mathrm{O}_{2}$, Earle's Balanced Salt Solution (EBSS) and serum starvation (SS) which can normally induce mitochondrial dysfunction. The results showed that HSD17B10 acetylation decreased, the level of SIRT3 expression increased, and the 


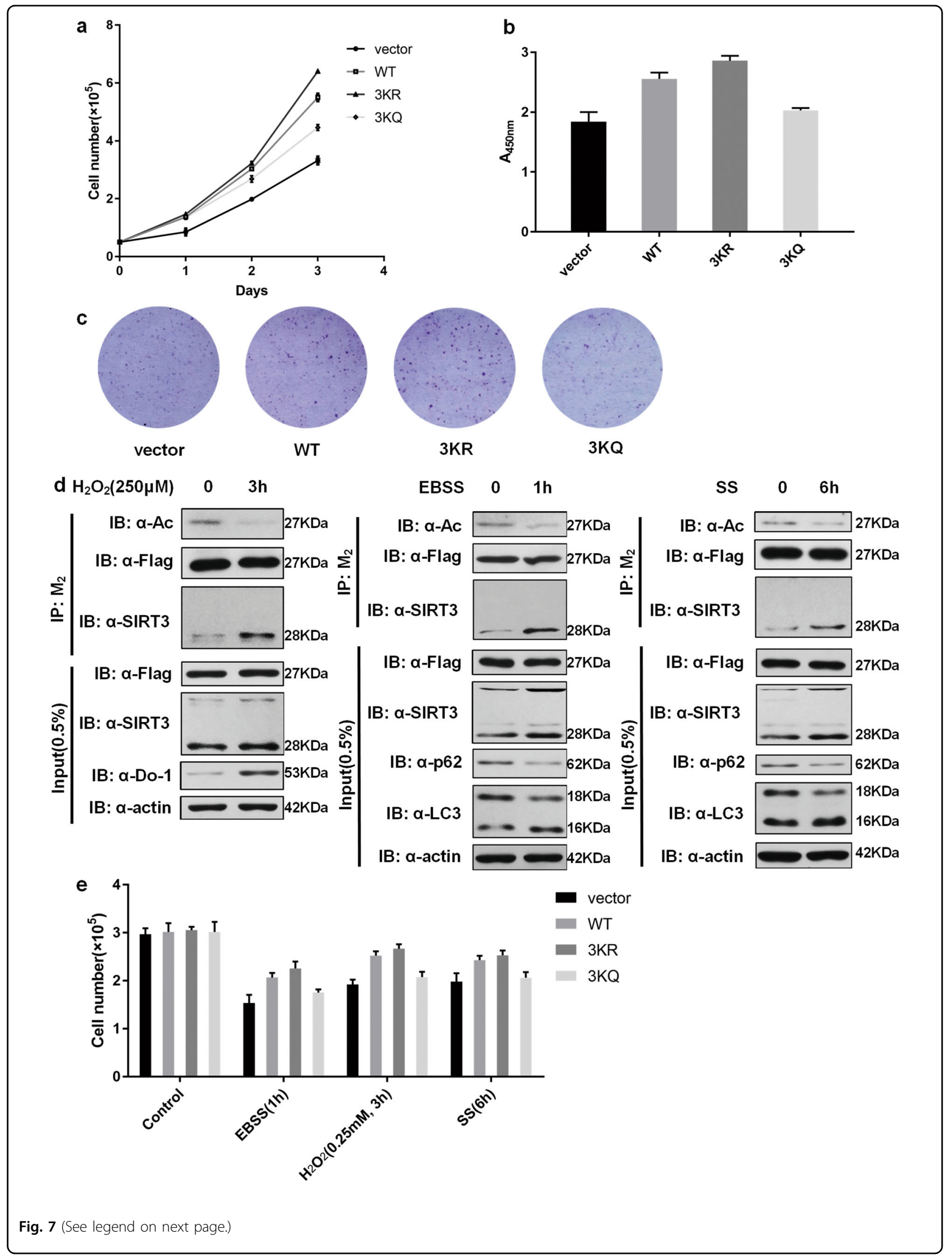


(see figure on previous page)

Fig. 7 HSD17B10 acetylation affects cell growth and cell resistance under stresses. a $5 \times 10^{4}$ HSD17B10 knockdown HCT116 re-expressed with vector, Flag-tagged HSD17B10 WT, 3KR and 3KQ cells were seeded into six-well plates. Cell number was counted every $24 \mathrm{~h}$. Data were shown as mean \pm S.D. $(n=3)$. b $5 \times 10^{3}$ HSD17B10 knockdown HCT116 re-expressed with vector, Flag-tagged HSD17B10 WT, 3KR and 3KQ cells were seeded into 96 -well plates. Cell growth was measured by CCK-8 assay after $48 \mathrm{~h}$. Data were shown as mean \pm S.D. $(n=3)$. c $5 \times 10^{3}$ HSD 17 B10 knockdown HCT116 re-expressed with vector, Flag-tagged HSD17B10 WT, 3KR and 3KQ cells were seeded into $6 \mathrm{~cm}$ plates. Colony formation was shown after crystal violet staining. $\mathbf{d}$ Flag-tagged HSD17B10 was transfected into HCT116 cells, and cells were treated with different stresses for the indicated time. HSD17B10 was IPed with $\mathrm{M}_{2}$ beads. e $2 \times 10^{5}$ HSD17B10 knockdown HCT116 re-expressed with vector, Flag-tagged HSD17B10 WT, 3KR and 3KQ cells were seeded into six-well plates. After $24 \mathrm{~h}$, cells were treated with different stresses for the indicated time, and the number of living cells was counted. Data were shown as mean \pm S.D. $(n=3)$.

interaction between HSD17B10 and SIRT3 became stronger under these treatments (Fig. 7d), demonstrating that SIRT3 could deacetylate HSD17B10 under stresses. Since HSD17B10 acetylation changed under stresses, we assumed that HSD17B10 acetylation might regulate cell resistance in response to stresses. We counted living cells after the treatments and verified that HSD17B10 knockdown cells lacked the resistance under stresses (Fig. S3d). With reexpression of HSD17B10 WT and 3KR, cell resistance was promoted under stresses compared to empty vector, while cells re-expressed with $3 \mathrm{KQ}$ were less resistant under stresses compared to WT (Fig. 7e). Taken together, HSD17B10 acetylation can affect cell growth and cell resistance in response to stresses.

\section{Discussion}

HSD17B10 is a member of short-chain dehydrogenase family as well as an essential subunit of human mitochondrial RNase P, an enzyme responsible for mitochondrial tRNA maturation. HSD17B10 is a highly conserved gene across a large evolutionary distance, and the missense mutations cause the loss of function in dehydrogenase activity and mitochondrial tRNA maturation, resulting in HSD10MD. HSD17B10 plays an important role in mitochondrial fatty acid metabolism and mitochondrial tRNA maturation. In this study, we identified HSD17B10 as a direct target of histone deacetylase SIRT3. Hypoacetylated HSD17B10 mediated by SIRT3 increased its enzymatic activity and mitochondrial RNase P formation. This regulation resulted in increased cell growth and cell resistance in response to stresses. Our study demonstrated that acetylation of HSD17B10 regulates its functions and results in a change of cellular phenotype.

HSD17B10 is a mitochondria-located protein and our data showed that NAM, a specific inhibitor of the sirtuins, enhanced its acetylation (Fig. 3a). SIRT3, SIRT4 and SIRT5 are three sirtuins reside within the mitochondria. Overexpression of SIRT3, SIRT4 and SIRT5 can all deacetylate HSD17B10 to different degrees (Fig. 3b). However, SIRT3 had the strongest deacetylation activity to HSD17B10. Yang et $\mathrm{al}^{26}$ found that there is an overlap in proteins associated with SIRT3, SIRT4 and SIRT5 and these three mitochondria sirtuins constitute the mitochondrial sirtuins network. Indeed, we also found that HSD17B10 exists in SIRT5 complex ${ }^{27}$. Additionally, SIRT4 could down-regulate
HSD17B10 (Fig. 3b), indicating that SIRT4 may also play an important role in regulating HSD17B10 functions. These observations may be worth further investigation regarding HSD17B10 function regulation.

It has been reported that HSD17B10 can be regulated by Parkin for mitochondrial abundance in a ubiquitindependent manner ${ }^{18}$. As acetylation can stabilize the protein by inhibiting its ubiquitination ${ }^{28}$, it is possible that HSD17B10 acetylation could affect its ubiquitination. However, our data did not implicate that acetylation can affect HSD17B10 abundance. It seems that these two posttranslational modifications do not have cross talk in regulating its functions; it could be that they are occurred at different lysine sites. Further exploration of other posttranslational modifications for HSD17B10 function regulation and their disease relevance would be worthwhile.

Missense mutations identified in HSD10MD cases have been verified to impair HSD17B10 dehydrogenase activity and mitochondrial RNase $\mathrm{P}$ function, thus impairing mitochondrial integrity and inducing apoptosis ${ }^{13,15,21}$. However, these mutations do not influence the acetylation of HSD17B10 (data not shown). HSD17B10 acetylation mutants 3KR (mimic for hypoacetylation status) and 3KQ (mimic for hyperacetylation status) were proved to affect its enzymatic activity and mitochondrial RNase P formation (Fig. 5e-g). As a consequence, HSD17B10 acetylation changes cell $\mathrm{NAD}^{+} / \mathrm{NADH}$ ratio and the amount of mt pretRNAs. Also, the mitochondrial membrane potential alters with the changes of HSD17B10 acetylation, indicating the dysfunction of mitochondria. It is possible that mutations in acetylation sites of HSD17B10 could lead to HSD10MD.

HSD17B10 may contribute to the neuronal dysfunction associated with Alzheimer disease (AD) by interacting with intracellular amyloid-beta $(A \beta)$, and binding with $A \beta$ can inhibit HSD17B10 activity ${ }^{17,29}$. Our data showed that acetylation of HSD17B10 can interrupt its interaction with TRMT10C to affect the formation of mt-tRNA splicing complex (Fig. 5f, g). As protein acetylation often affects protein-protein interaction, it will be interesting to investigate if acetylation of HSD17B10 can interfere the interaction of HSD17B10 and $A \beta$ to affect the development of AD.

Overexpression of HSD17B10 has been proved to increase cell growth and resistance to cell death ${ }^{22}$. As 
expected, HSD17B10 acetylation can regulate cell growth. SIRT3 is a stress-responsive deacetylase and we have proved that HSD17B10 acetylation plays a regulatory role in maintaining mitochondrial membrane potential. HSD17B10 acetylation levels decreased under stresses, and SIRT3 acts as the deacetylase. Deacetylated HSD17B10 protected cells from apoptosis under stresses comparing with acetylated HSD17B10 (Fig. 7d, e). Our study provides a possible mechanism for how HSD17B10 functions in cell growth and protecting cells from apoptosis. The results demonstrated that acetylation is an important regulation mechanism for HSD17B10 and may provide insight into interrupting the development of Alzheimer disease.

\section{Acknowledgements}

We thank Thomas Luo for editorial assistance and thank Qihua He (Peking University Health Science Center) for the confocal microscopy assistance. This work was supported by the National Natural Science Foundation of China (81874147, 81671389, and 81621063).

\section{Author details \\ 'Department of Medical Genetics, Center for Medical Genetics, Peking University Health Science Center, Beijing 100191, China. ${ }^{2}$ Department of Biochemistry and Molecular Biology, Thomas Jefferson University, 233 South 10th Street, 220 BLSB, Philadelphia, PA 19107, USA. ${ }^{3}$ Department of Biochemistry and Molecular Biology, Shenzhen University School of Medicine, Shenzhen 518060, China. ${ }^{4}$ Beijing Key Laboratory of Protein Posttranslational Modifications and Cell Function, Department of Biochemistry and Molecular Biology, Peking University Health Science Center, Beijing 100191, China}

\section{Conflict of interest}

The authors declare that they have no conflict of interest.

\section{Publisher's note}

Springer Nature remains neutral with regard to jurisdictional claims in published maps and institutional affiliations.

Supplementary Information accompanies this paper at (https://doi.org/ 10.1038/s41419-020-02763-9).

Received: 30 September 2019 Revised: 8 July 2020 Accepted: 9 July 2020 Published online: 23 July 2020

\section{References}

1. He, X. Y., Dobkin, C. \& Yang, S. Y. 17ß-Hydroxysteroid dehydrogenases and neurosteroid metabolism in the central nervous system. Mol. Cell. Endocrinol. 489, 92-97 (2019).

2. Moeller, G. \& Adamski, J. Integrated view on 17beta-hydroxysteroid dehydrogenases. Mol. Cell. Endocrinol. 301, 7-19 (2009).

3. He, X. Y. et al. Function of human brain short chain L -3-hydroxyacyl coenzyme A dehydrogenase in androgen metabolism. Biochim. Biophys. Acta 1484, 267-277 (2000).

4. Shafqat, N. et al. Expanded substrate screenings of human and Drosophila type 10 17beta-hydroxysteroid dehydrogenases (HSDs) reveal multiple specificities in bile acid and steroid hormone metabolism: characterization of multifunctional 3alpha/7alpha/7beta/17beta/20beta/21-HS. Biochem. J. 376, 49-60 (2003).

5. Yang, S. Y., He, X. Y. \& Schulz, H. 3-Hydroxyacyl-CoA dehydrogenase and short chain 3-hydroxyacyl-CoA dehydrogenase in human health and disease. FEBS $\mathrm{J}$. 272, 4874-4883 (2005).
6. Holzmann, J. et al. RNase P without RNA: identification and functional reconstitution of the human mitochondrial tRNA processing enzyme. Cell 135 462-474 (2008).

7. Leontis, N., Dalio, A., Strobel, M. \& Engelke, D. Effects of tRNA-intron structure on cleavage of precursor tRNAs by RNase P from Saccharomyces cerevisiae. Nucleic Acids Res. 16, 2537-2552 (1988).

8. Vilardo, E. et al. A subcomplex of human mitochondrial RNase $P$ is a bifunctional methyltransferase-extensive moonlighting in mitochondrial tRNA biogenesis. Nucleic Acids Res. 40, 11583-11593 (2012).

9. Helm, M. et al. The presence of modified nucleotides is required for cloverleaf folding of a human mitochondrial tRNA. Nucleic Acids Res. 26, 1636 (1998).

10. Reinhard, L., Sridhara, S. \& Hallberg, B. M. The MRPP1/MRPP2 complex is a tRNA-maturation platform in human mitochondria. Nucleic Acids Res. 45, 12469-12480 (2017).

11. Marques, A. T., Antunes, A., Fernandes, P. A. \& Ramos, M. J. Comparative evolutionary genomics of the $\mathrm{HADH} 2$ gene encoding Abeta-binding alcohol dehydrogenase/17beta-hydroxysteroid dehydrogenase type 10 (ABAD/ HSD10). BMC Genomics 7, 202 (2006).

12. Zschocke, J. HSD10 disease: clinical consequences of mutations in the HSD17B10 gene. J. Inherit. Metab. Dis. 35, 81-89 (2012).

13. Falk, M. J. et al. A novel HSD17B10 mutation impairing the activities of the mitochondrial RNase $\mathrm{P}$ complex causes $\mathrm{X}$-linked intractable epilepsy and neurodevelopmental regression. RNA Biol. 13, 477-485 (2016).

14. Oerum, S. et al. Novel patient missense mutations in the HSD17B10 gene affect dehydrogenase and mitochondrial tRNA modification functions of the encoded protein. Biochimica et biophysica acta. Mol. Basis Dis. 1863 3294-3302 (2017)

15. Ofman, R. et al. 2-methyl-3-hydroxybutyryl-CoA dehydrogenase deficiency is caused by mutations in the HADH2 gene. Am. J. Hum. Genet. 72, 1300-1307 (2003).

16. Lustbader, J. W. et al. ABAD directly links Abeta to mitochondrial toxicity in Alzheimer's disease. Science 304, 448-452 (2004).

17. Oppermann, U. C., Salim, S., Tjernberg, L. O., Terenius, L. \& Jörnvall, H. Binding of amyloid beta-peptide to mitochondrial hydroxyacyl-CoA dehydrogenase (ERAB): regulation of an SDR enzyme activity with implications for apoptosis in Alzheimer's disease. FEBS Lett. 451, 238 (1999).

18. Bertolin, G. et al. Parkin maintains mitochondrial levels of the protective Parkinson's disease-related enzyme 17-beta hydroxysteroid dehydrogenase type 10. Cell Death Differ. 22, 1563-1576 (2015).

19. Chen, S. et al. SIRT3 regulates cancer cell proliferation through deacetylation of PYCR1 in proline metabolism. Neoplasia 21, 665-675 (2019).

20. Binstock, J. F. \& Schulz, H. Fatty acid oxidation complex from Escherichia coli. Methods Enzymol. 71, 403-411 (1981).

21. Rauschenberger, K. et al. A non-enzymatic function of 17beta-hydroxysteroid dehydrogenase type 10 is required for mitochondrial integrity and cell survival. EMBO Mol. Med. 2, 51-62 (2010).

22. Carlson, E. A. et al. Overexpression of 17beta-hydroxysteroid dehydrogenase type 10 increases pheochromocytoma cell growth and resistance to cell death. BMC Cancer 15, 166 (2015).

23. Hirschey, M. D. et al. SIRT3 regulates mitochondrial fatty-acid oxidation by reversible enzyme deacetylation. Nature 464, 121-125 (2010).

24. Jing, E. et al. Sirtuin-3 (Sirt3) regulates skeletal muscle metabolism and insulin signaling via altered mitochondrial oxidation and reactive oxygen species production. Proc. Natl. Acad. Sci. USA 108, 14608-14613 (2011).

25. Sundaresan, N. R., Samant, S. A., Pillai, V. B., Rajamohan, S. B. \& Gupta, M. P. SIRT3 is a stress-responsive deacetylase in cardiomyocytes that protects cells from stress-mediated cell death by deacetylation of Ku70. Mol. Cell. Biol. 28, 6384-6401 (2008)

26. Yang, W. et al. Mitochondrial sirtuin network reveals dynamic SIRT3dependent deacetylation in response to membrane depolarization. Cell 167, 985-1000.e1021 (2016).

27. Yang, X. et al. SHMT2 desuccinylation by SIRT5 drives cancer cell proliferation. Cancer Res. 78, 372-386 (2018).

28. Li, K. et al. Acetylation of WRN protein regulates its stability by inhibiting ubiquitination. PLOS ONE 5, e10341 (2010).

29. Yan, S. D. et al. An intracellular protein that binds amyloid-beta peptide and mediates neurotoxicity in Alzheimer's disease. Nature 389, 689-695 (1997). 